\title{
Os desafios do design centrado no usuário para validação de um sistema informatizado em Universidade Pública
}

\author{
The challenges of user-centered design for validation of a computerized system at \\ Public University
}

MARTINS, Anny Caroline Castelo Branco; Bacharel em Design; UFAM

annycarolinecb@gmail.com

FALCÃO, Franciane da Silva; Doutora em Design; UFAM

francifalcao@gmail.com

\section{Resumo}

O Programa Acadêmico de Monitoria, da Universidade Federal do Amazonas (UFAM), exige um processo de gerenciamento robusto. Um módulo para sistema informatizado de gerenciamento acadêmico, já existente, e a adequação da norma atual, foi o caminho encontrado para solucionar problemas de: aplicação diferenciada dos processos operacionais entre as unidades acadêmicas, distância geográfica entre gestor e sedes de outros municípios, atraso no cumprimento de prazos, falta de informação aos distintos grupos de usuários, deficiência na emissão e recebimento de documentações. O desafio para validação deste módulo informatizado conduziu à revisão sobre as técnicas da área de design centrado no usuário, e busca de recursos para aplicação de testes remotos devido à dificuldade geográfica de acesso aos usuários. Os recursos disponíveis de uma plataforma em web e a representatividade geográfica de usuários participantes indicam estes testes como uma boa alternativa para superar dificuldades de acesso ao público e minimizar custos de aplicação.

Palavras Chave: testes remotos; design centrado no usuário; informatização de processos.

\begin{abstract}
The Academic Monitoring Program of the Federal University of Amazonas (UFAM) requires a robust management process. A module for an existing computerized academic management system and the adequacy of the current standard was the way of solving the problems of: differentiated application of operational processes between academic units, geographical distance between manager and headquarters of other municipalities, delay in compliance with deadlines, lack of information to the different groups of users, deficiency in the issuance and receipt of documentation. The challenge for validation of this computerized module led to a review of user-centered design techniques and the search for resources for the application of remote tests due to the geographical difficulty of access to users. The available resources of a web platform and the geographical representativeness of participating users indicate these tests as a good alternative to overcome difficulties of access to the public and to minimize application costs.
\end{abstract}

Keywords: remote testing; user-centered design; computerization of processes. 


\section{Introdução}

As atividades de ensino, pesquisa e extensão são inerentes à universidade e isto a diferencia das demais instituições de ensino superior (MEC, 2018). Gomes (2010) define as universidades como instituições que agregam inúmeras áreas de conhecimento para formar profissionais de nível superior. O autor ainda acrescenta que "é constituída por um conjunto de unidades (centros, institutos, faculdades, departamentos, laboratórios e núcleos). Confere graus, diplomas e títulos como os de bacharel, licenciado, especialista, mestre e doutor" (GOMES, 2010, p. 593 apud CAMPOS; MACHADO, 2000, p. 350-351).

De acordo com a descrição de Gomes (2010) pode-se perceber a complexidade de informações e a quantidade de pessoas que transitam em uma universidade. Essa afirmação pode ser confirmada ao analisar os dados divulgados pela Universidade Federal do Amazonas (UFAM) por meio da Pró-reitora de Planejamento (PROPLAN, 2014), são cerca de 20 unidades acadêmicas, sendo 15 unidades localizadas na capital e 5 no interior do Amazonas nas cidades de Parintins, Humaitá, Benjamin Constant, Coari e Itacoatiara. De acordo com os dados referentes ao segundo semestre do ano de 2014, a UFAM possui 52.163.276,72 $\mathrm{m}^{2}$ de área territorial, 18.036 alunos matriculados na graduação e 1.714 docentes. Portanto, diante desses números é notável a necessidade de gerenciamento, organização e planejamento dos processos executados dentro da universidade.

$\mathrm{Na}$ Universidade Federal do Amazonas são ofertados inúmeros programas que atendem todos os campis, incluindo capital e interior do Estado. Um deles é o programa de monitoria que é considerado uma importante ferramenta que visa inserir os alunos na carreira docente. Por meio deste programa que os discentes têm a oportunidade de conhecer na prática quais são as técnicas, formas de planejamento e controle para ensinar. Nos últimos anos houve um aumento na procura pelo programa e são cadastrados cerca de 900 monitores, esses cadastros são feitos manualmente e gerenciados por uma técnica administrativa, gerando cerca de 5.400 documentações semestralmente (MARTINS, 2017).

O programa de monitoria é regulado pela resolução 006/2013 CONSEPE- CEG, porém pela falta de fiscalização e incoerência das regras com a realidade vivenciada pelos usuários, a resolução é pouco aplicada. Cada unidade realiza a monitoria da forma mais conveniente gerando vários problemas. $O$ edital exigido na resolução é executado por menos de $50 \%$ das unidades acadêmicas, que fazem a divulgação pelas redes sociais, e-mails ou não realizam o processo seletivo. Essa disparidade de ações reflete na vida de todos os envolvidos, principalmente na coordenação do programa, cuja gerente tem uma carga de trabalho extensa e não possui tempo para melhorar os processos de gerenciamento, conseguir mais recursos ou até mesmo fiscalizar o cumprimentos das normas.

Um estudo realizado por Martins (2017), propôs inserir o programa de monitoria como um módulo dentro do sistema de gerenciamento acadêmico utilizado pela universidade: o ecampus. 0 desenvolvimento da proposta de digitalização dos processos do programa de monitoria foi realizado por meio da abordagem de design centrado no usuário, porque envolvia um público diverso de alunos, professores, setores e gestores localizados em diferentes cidades do Estado do Amazonas. Durante a projetação surgiram obstáculos que expuseram as dificuldades de aplicação da abordagem do design centrado no usuário neste cenário institucional robusto e intermunicipal. Isto se transformou em insumo para pensar em novas formas de projetar. Neste artigo serão abordados 
os desafios e soluções encontradas ao aplicar as técnicas de card sorting e medição de performance, utilizadas durante o desenvolvimento do módulo de monitoria para o ecampus.

\section{Design centrado no usuário e suas técnicas}

Norman (2006) defende que o Design Centrado no Usuário (User Centered Design - UCD) ou Design Centrado no Ser Humano (Human Centered Design -HCD) seja uma filosofia para projetar produtos compreensíveis e facilmente utilizáveis. O design deve tornar as tarefas mais fáceis e por isso deve-se fazer uso de características inerentes das pessoas e do mundo. Dessa forma são os usuários que ditam as necessidades para que os designers encontrem a solução. Shibuya (2010) ressalta que para o HCD seja legítimo, durante o processo de design (seja qual for a fase) o usuário deve participar.

A Ideo (2009) cita estudos de casos em que o design centrado no usuário foi imprescindível para alcançar o sucesso e impactar na vida de milhões de pessoas. Entre eles está o caso da positivo, que criou o computador a baixo custo, a mudança estratégica da Havaianas que a permitiu explorar novos nichos de mercado e o sistema desenvolvido para doação de sangue da Cruz Vermelha. Na percepção de Kalbach (2009, p.39) "ao considerar cuidadosamente o real contexto de uso antes que um produto seja lançado no mercado, o design centrado no usuário aumenta potencialmente as taxas de adoção e diminui a curva de aprendizagem." Receber feedbacks antes de implementar é a principal vantagem de projetar com o auxílio do usuário porque economiza tempo e dinheiro.

No mercado existem técnicas de design centrado no usuário voltadas para cada etapa projetual, foram escolhidas algumas delas para detalhar: entrevista e questionários, card sorting e teste de usabilidade e medição de performance.

\subsection{Entrevista e questionários}

As entrevistas e os questionários são formas de definir o que será o produto e se este planejamento está de acordo com as expectativas dos envolvidos no projeto. Entrevistar é a mesma coisa que conversar com o usuário, para Freire (2005) apud Marconi e Lakatos (1990) vale ressaltar a necessidade de pautar a entrevista para que o entrevistador consiga obter informações importantes acerca do problema do usuário. Da mesma forma, são os questionários que precisam ser claros e específicos, visto que nem sempre o pesquisador estará ao lado do usuário para esclarecer dúvidas. Ambas as técnicas são difundidas e utilizadas em várias situações e em momentos diferentes do projeto, geralmente são as entrevistas que pautam o início do projeto e os questionários são utilizados após a implementação afim de entender o grau de satisfação do usuário (FREIRE, 2005).

\subsection{Card sorting}

O card sorting consiste em reunir e pedir para que os usuários agrupem informações e funções em categorias, ajuda os designers a compreender a lógica e o entendimento dos usuários sobre o sistema (TEXEIRA, 2015). É uma técnica que pode ser utilizada no projeto de um novo site, na criação de uma nova área do site e no redesign de um site (PIRAUÁ, MOURA e PADOVANI, 2006 apud MAURER e WARFEL, 2005). O card sorting pode ser dividido entre: aberto ou fechado e de baixa ou alta fidelidade, o aberto o usuário fica livre para agrupar enquanto no fechado os grupos são previamente criados e rotulados pelo pesquisador. Quanto a fidelidade está relacionada ao meio utilizado, papel para o de baixa fidelidade e softwares para o de alta fidelidade. 


\subsection{Teste de usabilidade e medição de performance}

"Testes de usabilidade são experimentos realizados por pesquisadores afim de investigar as ações e comportamentos dos usuários potenciais do sistema" (FREIRE, 2005, p.283 apud DIAS, 2003). E de acordo com a mesma autora a medição de performance são similares aos testes de usabilidade, porém, tem como foco a captura de dados quantitativos e qualitativos, como tempo de clique, erros cometidos no processo, consultas ao tópico de ajuda do sistema e opinião dos usuários. Os testes de usabilidade podem ser controlados ou em contexto real de uso: O primeiro é realizado em laboratórios com auxílio de equipamentos de gravação, o segundo no ambiente familiar ao usuário. Nielsen (2002) afirma que em testes controlados o usuário pode não se sentir à vontade devido ao ambiente de teste artificial e nas abordagens em contexto real nem sempre é possível controlar as adversidades.

Os testes de usabilidade são indicados para serem realizados em qualquer fase do projeto, já os testes de performance são mais viáveis para a etapa final de projetação, para indicar pontos onde o usuário pode ter se deparado com erros, ou com alguma dificuldade que o impediu ou atrasou de completar a tarefa. A importância de ambos é a obtenção de feedbacks que auxiliem os projetistas a pensar novas soluções, identificar problemas e tornar a interface mais amigável a quem utiliza.

Com as três técnicas citadas é possível envolver o usuário no projeto do início ao fim, proporcionando empatia entre projetistas e usuários. As entrevistas e questionários são a base para conhecer o problema e definir o perfil dos usuários, o card sorting ajuda a compreender o agrupamento e organização que os usuários fazem do sistema e o teste de usabilidade é a principal forma de medir e avaliar quão eficiente é o sistema, por meio da observação da reação e interação dos usuários com produto.

Portanto, o design centrado no usuário é uma forma de garantir que o projeto a ser desenvolvido esteja alinhado com as necessidades dos usuários e tenha uma curva de aprendizagem menor. Estes fatores são essenciais quando o público do projeto apresenta grande diversidade como é o caso de projetos realizados dentro de uma universidade, que deve atender alunos, professores e servidores de forma ágil e rápida sem demandar uma grande carga de aprendizagem.

\section{Materiais e Métodos}

O desenvolvimento de uma proposta, de modulo informatizado, de gerenciamento para Programa de Monitoria, no sistema ecampus, foi norteado pela metodologia Projeto E (MEURER e SZABLUK, 2012), que é composto por seis etapas de desenvolvimento: estratégia, escopo, estrutura, esqueleto, estética e execução (Figura 01). Os principais desafios encontrados impactaram diretamente na aplicação das técnicas de entrevista, card sorting, e medição de performance, que integraram respectivamente as etapas de estratégia, escopo e estrutura, execução. 
Figura 1 - Etapas da Metodologia do projeto $\mathrm{E}$

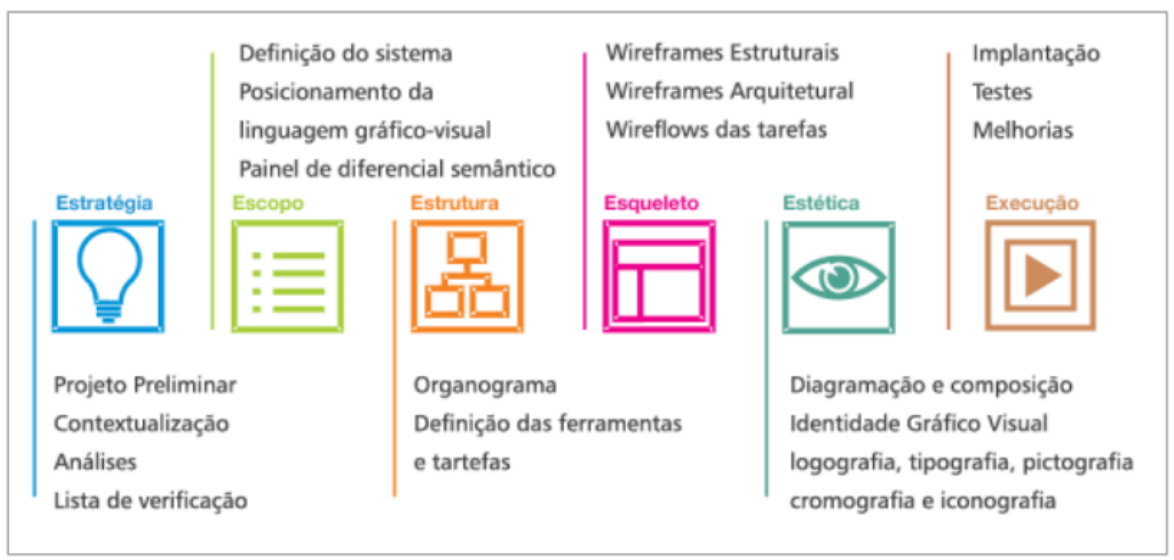

Fonte: MEURER e SZABLUK (2012)

Antes de definir a forma de aplicação das técnicas supracitadas, no contexto institucional da UFAM, foi necessário a descrição do público diverso de alunos, professores, setores e gestores, e as distintas funções que assumem no processo de monitoria (Quadro 1). Para tanto, foi realizada pesquisa documental a partir do estudo da Resolução n0 006/2013 CEG/CONSEPE (2013) e formulários institucionais do Programa de Monitoria, por meio do qual foi identificada os agentes do processo e suas funções (Quadro 1), bem como o fluxo de ações preconizados pela norma, que não é seguida por todos os setores da universidade. A partir disto foi possível caracterizar os grupos de sujeitos com os quais seria necessário coletar informações.

Quadro 1 - Funções da cada um do agentes dos Processos de Monitoria.

\begin{tabular}{|c|c|c|c|c|}
\hline Monitor & Professor & $\begin{array}{l}\text { Coordenador da } \\
\text { Monitoria no Setor } \\
\text { (Departamento) }\end{array}$ & $\begin{array}{l}\text { Comissão de } \\
\text { Monitoria }\end{array}$ & $\begin{array}{l}\text { Gerente da } \\
\text { Monitoria na Pró- } \\
\text { reitora de } \\
\text { Graduação (PROEG) }\end{array}$ \\
\hline $\begin{array}{l}\text { Apoiar professor e } \\
\text { alunos da disciplina, } \\
\text { constituindo um elo } \\
\text { entre eles. }\end{array}$ & $\begin{array}{l}\text { Estimular, orientar e } \\
\text { avaliar o } \\
\text { desenvolvimento das } \\
\text { atividades } \\
\text { produzidas pelo } \\
\text { monitor. }\end{array}$ & $\begin{array}{l}\text { Divulgar e gerenciar } \\
\text { o processo de } \\
\text { monitoria no âmbito } \\
\text { do departamento. }\end{array}$ & $\begin{array}{l}\text { Coordenar, avaliar e } \\
\text { deliberar a } \\
\text { distribuição de vagas } \\
\text { para o programa de } \\
\text { monitoria. }\end{array}$ & $\begin{array}{l}\text { Administrar, definir, } \\
\text { informar, organizar, } \\
\text { elaborar } \\
\text { documentações } \\
\text { relativas ao } \\
\text { programa de } \\
\text { monitoria. }\end{array}$ \\
\hline
\end{tabular}

Fonte: produzido pelos autores (2018)

Assim foram estabelecidas as finalidades para aplicação das técnicas, os sujeitos, recursos necessários e procedimentos de cada técnica aplicada. Abaixo (Quadro 2) apresentamos esta descrição referente as três técnicas abordadas neste artigo (entrevista, card sorting, e medição de performance). 
Quadro 2 - Descrição dos procedimentos de aplicação das técnicas

\begin{tabular}{|c|c|c|c|c|c|}
\hline 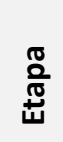 & Técnica & Finalidade & $\begin{array}{l}\text { Características dos } \\
\text { sujeitos }\end{array}$ & Recursos & Procedimentos \\
\hline & $\begin{array}{l}\text { Entrevistas } \\
\text { não } \\
\text { estruturada }\end{array}$ & $\begin{array}{l}\text { Entender como o } \\
\text { processo acontece nos } \\
\text { setores, o contexto e o } \\
\text { problema de forma } \\
\text { preliminar. }\end{array}$ & $\begin{array}{l}1 \text { coordenadora de } \\
\text { monitoria no setor, } \\
\text { com mais de } 2 \text { anos } \\
\text { de atuação no } \\
\text { programa. }\end{array}$ & $\begin{array}{l}\text { bloco de } \\
\text { anotação e } \\
\text { caneta }\end{array}$ & $\begin{array}{l}\text { Perguntas abertas } \\
\text { emitidas sobre a } \\
\text { prática de } \\
\text { coordenação do } \\
\text { programa e principais } \\
\text { problemas } \\
\text { identificados na } \\
\text { prática. }\end{array}$ \\
\hline 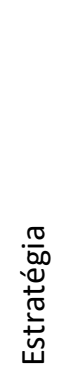 & $\begin{array}{l}\text { Entrevista } \\
\text { estruturada }\end{array}$ & $\begin{array}{l}\text { Entender como o } \\
\text { processo funciona } \\
\text { atualmente, as } \\
\text { atividades de } \\
\text { gerenciamento na } \\
\text { reitoria e os problemas } \\
\text { do programa de } \\
\text { monitoria. }\end{array}$ & $\begin{array}{l}1 \text { gerente de } \\
\text { monitoria na } \\
\text { PROEGA (único } \\
\text { sujeito que possui } \\
\text { esta função na } \\
\text { instituição); com } \\
\text { mais de } 2 \text { anos de } \\
\text { atuação no } \\
\text { programa. }\end{array}$ & $\begin{array}{l}\text { Gravador, bloco } \\
\text { de anotação e } \\
\text { caneta }\end{array}$ & $\begin{array}{l}\text { Elaborar roteiro } \\
\text { perguntas; } \\
\text { Marcar reunião com } \\
\text { a gerente; } \\
\text { Realizar as perguntas } \\
\text { na data e horário } \\
\text { marcado. }\end{array}$ \\
\hline 욤 & $\begin{array}{l}\text { Card sorting } \\
\text { de baixa } \\
\text { fidelidade } \\
\text { (papel) }\end{array}$ & $\begin{array}{l}\text { Definir a navegação e } \\
\text { os rótulos do sistema }\end{array}$ & $\begin{array}{l}4 \text { alunos, } 3 \\
\text { professores, } 2 \\
\text { setores e } 1 \text { gerente, } \\
\text { que participaram da } \\
\text { monitoria alguma } \\
\text { vez. }\end{array}$ & $\begin{array}{l}\text { Cartões } \\
\text { coloridos, } \\
\text { canetas, lápis e } \\
\text { borracha }\end{array}$ & $\begin{array}{l}\text { Preparar cartões com } \\
\text { base nas } \\
\text { funcionalidades } \\
\text { definidas. } \\
\text { Convidar usuários } \\
\text { para participar. } \\
\text { Pedir que eles } \\
\text { agrupem os cartões. } \\
\text { Registrar as } \\
\text { disposições dos } \\
\text { cartões. }\end{array}$ \\
\hline 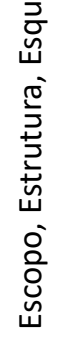 & $\begin{array}{l}\text { Card Sorting } \\
\text { de alta } \\
\text { fidelidade } \\
\text { (computador) }\end{array}$ & $\begin{array}{l}\text { Definir a navegação e } \\
\text { os rótulos do sistema }\end{array}$ & $\begin{array}{l}10 \text { alunos, } 7 \\
\text { professores e } 7 \\
\text { setores que } \\
\text { participaram da } \\
\text { monitoria alguma } \\
\text { vez. }\end{array}$ & $\begin{array}{l}\text { Plataforma Web } \\
\text { Optimal } \\
\text { Workshop }\end{array}$ & $\begin{array}{l}\text { Criar cartões virtuais } \\
\text { para as } \\
\text { funcionalidades } \\
\text { definidas. } \\
\text { Enviar procedimentos } \\
\text { e link por email. } \\
\text { Analisar resultados. }\end{array}$ \\
\hline
\end{tabular}




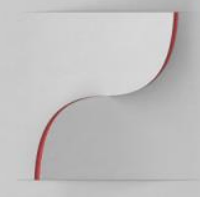

Pesquisa \&

Desenvolvimento

em Design

\section{Artigo Completo}

\begin{tabular}{|c|c|c|c|c|c|}
\hline$\frac{\pi}{2}$ & $\begin{array}{l}\text { Técnica } \\
\text { (CONT.) }\end{array}$ & Finalidade & $\begin{array}{l}\text { Características dos } \\
\text { sujeitos }\end{array}$ & Recursos & Procedimentos \\
\hline 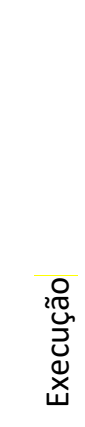 & $\begin{array}{l}\text { Medição de } \\
\text { performance }\end{array}$ & $\begin{array}{l}\text { Avaliar a interface } \\
\text { segundo os critérios de } \\
\text { usabilidade }\end{array}$ & $\begin{array}{l}106 \text { alunos, } 34 \\
\text { professores, } 22 \\
\text { setores e } 2 \\
\text { gerentes. Todos os } \\
\text { usuários afirmaram } \\
\text { conhecer ou ter } \\
\text { participado da } \\
\text { monitoria. }\end{array}$ & $\begin{array}{l}\text { Adobe } \\
\text { Photoshop CC } \\
2017, \\
\text { plataforma web } \\
\text { Optimal } \\
\text { Workshop e } \\
\text { Microsoft Office } \\
\text { Excel }\end{array}$ & $\begin{array}{l}\text { Definir as atividades } \\
\text { a serem testadas. } \\
\text { Listar todas as tarefas } \\
\text { e desenhar as telas. } \\
\text { Cadastrar as } \\
\text { atividades no Optimal } \\
\text { Workshop. } \\
\text { Compartilhar o link } \\
\text { do teste. } \\
\text { Analisar os resultados }\end{array}$ \\
\hline
\end{tabular}

Fonte: produzido pelos autores (2018)

\section{Desafios encontrados e Soluções Testadas}

Assim como toda pesquisa se depara com desafios, a utilização das técnicas de design centrado no usuário traz um agravante ainda maior: a participação efetiva dos usuários durante a projetação. Com a realização das técnicas de card sorting e medição de performance foi possível identificar claramente quais são as principais dificuldades de implementar esta metodologia em uma universidade pública.

\subsection{Participação dos envolvidos}

$\mathrm{Na}$ universidade existem diversos grupos: professores, coordenação, alunos e técnicos com rotinas totalmente diferentes, este fator contribui para dificultar a participação desses usuários. Para sessões presenciais é necessário encaixar data, horário e local que estejam favoráveis tanto para o pesquisador quanto ao usuário. Ao realizar a técnica de card sorting de baixa fidelidade, utilizando cartões impressos, notou-se a limitação de participantes. Em 9 dias de realização das sessões de card sorting somente 10 pessoas participaram, sendo representantes das três unidades Instituto de Filosofia, Ciências Humanas e Sociais (IFCHS), Instituto de Computação (ICOMP) e Faculdade de Tecnologia (FT).

A falta de comunicação coletiva entre membros pertencentes à monitoria foi um aspecto que contribuiu significativamente para restringir o público, dessa forma a pesquisa ficou dependente de grupos de amizade e redes sociais para ter contato com os usuários do programa de monitoria. Isto fica explícito quando se observa que das três unidades que tiveram participação, todas pertenciam ao setor norte do campus sede da universidade, evidenciando também a dificuldade de atingir os usuários de outros campis fora da sede de Manaus.

\subsection{Barreira Geográfica}

A Universidade Federal do Amazonas possui 20 unidades acadêmicas espalhadas pelo Estado do Amazonas, enfrentando grandes distâncias fluviais, como a de $1.575 \mathrm{~km}$ da capital amazonense até o município de Benjamin Constant (ASSOCIAÇÃO AMAZONENSE DE MUNÍCIPIOS, 2018). Além disso, mesmo os campis na cidade de Manaus são distantes um do outro e possuem dinâmicas 
diferentes.

Diante deste cenário a realização de testes presenciais com estes usuários tornam-se inviáveis do ponto de vista geográfico e financeiro, para não excluir este público foi necessário articular e adaptar as técnicas tradicionais de design centrado no usuário.

\subsection{Tempo de execução da pesquisa}

Os prazos são primordiais para conseguir alcançar os resultados esperados em qualquer pesquisa, porém ao optar por trazer os usuários para contribuir com o processo é preciso ter em mente um bom planejamento. As sessões de card sorting presenciais demonstraram quão difícil é conseguir a disponibilidade total do usuário para a realização de uma atividade de duração média de 15 minutos. Apesar de ser uma atividade rápida, o card sorting precisa de atenção e concentração, foi notado que durante algumas sessões os usuários demonstraram precipitação nas suas escolhas e deixaram de dar sugestões por não querer demorar mais que a média.

Levando em consideração os números obtidos, foi realizada aproximadamente 1 sessão por dia durante 9 dias. Este dado é relevante para explicitar quão demorado pode ser aplicar testes com a presença dos usuários fisicamente, porque assim como o desenvolvimento do sistema de monitoria, não foi realizado testes com somente uma técnica, foram um conjunto de ferramentas utilizadas em cada etapa da projetação. $O$ atraso em qualquer uma das etapas pode prejudicar o andamento da pesquisa e por isso optou-se por utilizar uma plataforma digital que resolvesse a comunicação com os usuários, a barreira geográfica e a limitação de tempo de execução.

\subsection{Análise e comparação dos resultados obtidos por meio das técnicas.}

Após a execução das técnicas e tabulação dos resultados, utilizou-se o software Microsoft Excel para comparar estatisticamente e gerar gráficos comparativos entre as técnicas. Essa comparação permitiu evidenciar qual forma de execução que trouxe maior participação dos usuários e artifícios usados para engajar os usuários.

O primeiro aspecto identificado foi a apresentação das instruções que os usuários deviriam fazer, expondo de forma clara e objetiva o propósito da atividade e qual o benefício atrelado a ela. Para isso foi feito pequenos infográficos, como na figura 2, com as funcionalidades que a plataforma iria oferecer a cada usuário, evidenciando quão importante é a participação dele para a construção do sistema. Foi produzido um vídeo ${ }^{1}$ explicando o que era a técnica do card sorting, o objetivo e como funcionava o teste na plataforma. Por email foram enviados os convites explicando a motivação da pesquisa e o roteiro do teste.

De imediato houve um feedback do público mostrando interesse em ajudar, demonstrando que eles se sentiram engajados e motivados com a pesquisa. Isso foi essencial para ter um resultado fiel ao que os usuários pensam e quais soluções eles queriam para o problema. Não houveram dúvidas quanto à realização o teste ou sobre a técnica o que significa que os participantes conseguiram absorver as informações facilmente.

\footnotetext{
${ }^{1}$ Acesse o vídeo: https://www.youtube.com/watch?v=n6HSrMPA8eo
} 
Figura 2 - Infográfico

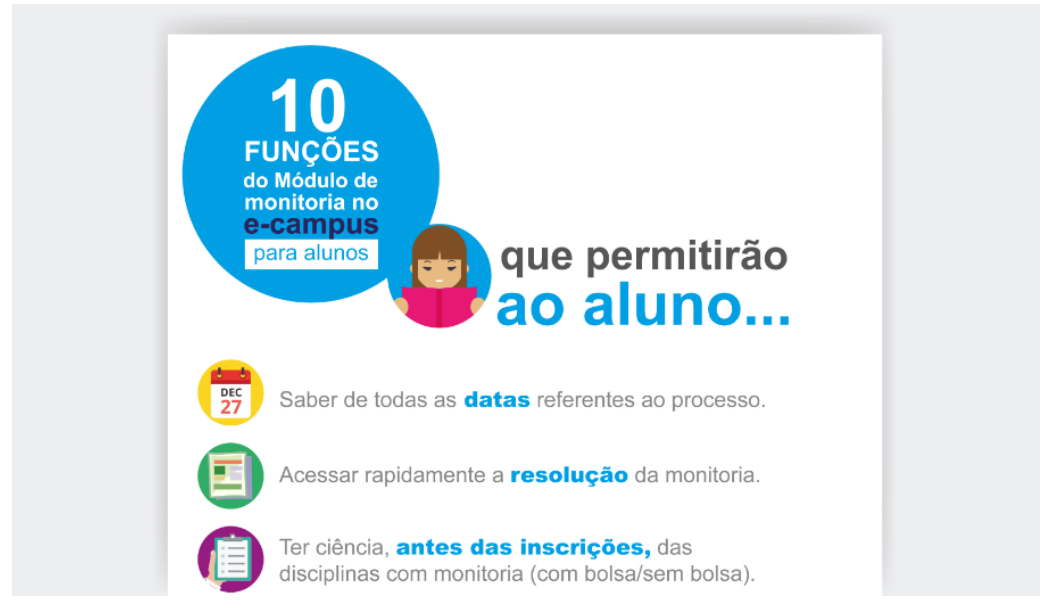

Fonte: Martins (2017)

A aplicação das sessões de card sorting online serviram de aprendizado para buscar mais formas de fazer com que os usuários se engajassem. Por isso ao finalizar a criação do sistema e aplicar o teste de performance foi oferecido um certificado de participação valendo horas complementares. Somando a quantidade de resposta do card sorting presencial e online em relação às respostas da medição de performance, constatou-se um aumento a participação dos usuários 4,8 vezes maior como mostra a figura 3.

Figura 3 - Comparação da quantidade de participantes

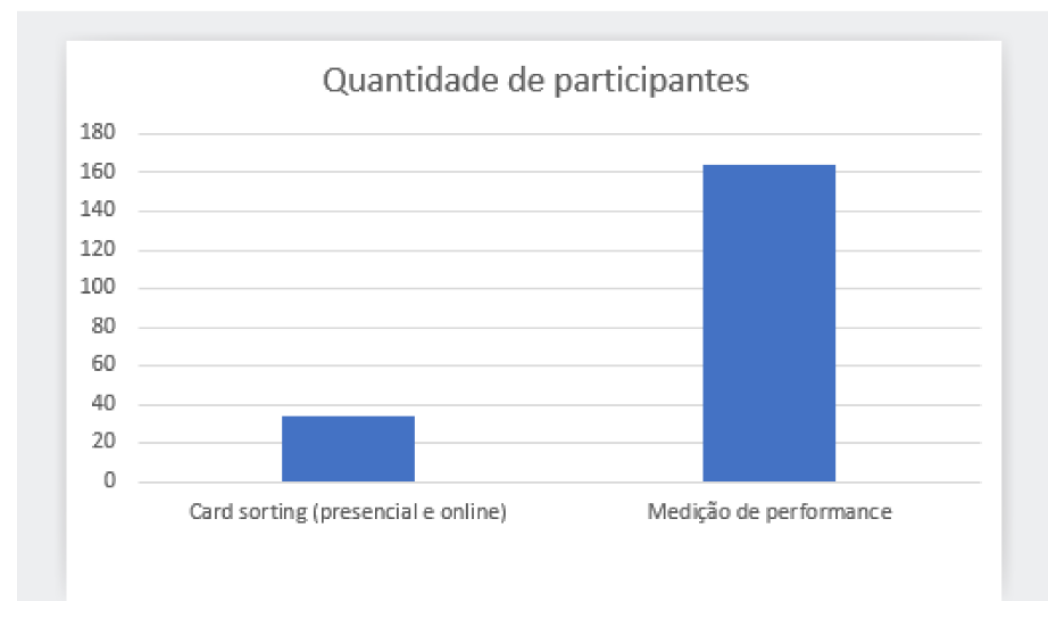

Fonte: Martins (2017)

Apesar das técnicas não terem sido aplicadas sobre as mesmas condições, a medição de performance foi totalmente online enquanto o card sorting teve uma parte presencial, o tempo para a realização de ambas foi de 23 dias. Criando hipoteticamente outro cenário, em que o card sorting teria sido realizado totalmente online e mantendo a mesma média de participantes obtidos (24 participantes em 14 dias) durante 23 dias, os resultados poderiam ser de 39 participantes, 15 a mais que o resultado alcançado. Ou seja, isso demonstra que não somente o uso de mecanismos de recompensas ajudou nos resultados, mas também o uso da plataforma digital foi um fator determinante para alcançar um número maior de participantes. 
Em 14 dias de realização das sessões de card sorting online conseguiram-se 24 participantes. Mesmo não sendo um número exageradamente maior que os testes presenciais, houve a participação de alunos, professores e coordenadores das cidades do interior e de outras unidades em Manaus. O card sorting online e presencial somaram um total de 34 participantes, sendo que online foi possível atingir 11 unidades a mais que nos testes presenciais, incluindo duas unidades do interior do Amazonas.

Outro aspecto, além da barreira geográfica, foi a rapidez em que se conseguiu testar com um grande número de usuários. A medição de performance foi aplicada totalmente online e atingiu uma média de 7 participantes por dia. Com a ajuda da plataforma foi possível analisar a eficácia, eficiência e satisfação reunindo um conjunto de dados, como tempo de clique, erros, acertos e opinião que se transformaram em insumo para tomar decisões de mudança no layout. Esses mesmos aspectos sendo analisados por meio de testes de usabilidade tradicionais necessitariam de mais tempo e recurso.

De modo geral foi demonstrado que não há impedimentos para a execução de pesquisas com design centrado no usuário em cenários onde o público é amplo e disperso, existem dificuldades que podem ser resolvidas de acordo com a limitação de cada projeto. A utilização da plataforma trouxe ganhos significativos e mostrou a importância da utilização de meios eficientes de engajar usuários e obter dados quantitativos nos testes de interface.

\section{Considerações Finais}

Temos no mercado diversas plataforma em web que possibilitam simulações de testes de distintas técnicas da área do design centrado no usuário. A escolha da plataforma deve ser norteada pelas funções e atividades necessários ao teste planejado, o número de participantes que se almeja atingir, bem como o nível de realismo que a simulação pode proporcionar. As principais vantagens destas plataformas estão na possibilidade de atingir usuários que estão há consideráveis distancias geográficas a um baixo custo operacional, gerar relatórios de resultados obtidos pelo teste que são compatíveis com arquivos Excel, conferir aos sujeitos do teste o conforto de fazer o teste em seu ambiente típico de uso do sistema informatizado testado.

As funções específicas disponíveis na plataforma em web Optimal Workshop ${ }^{2}$, que levaram a escolha da mesma são: a usabilidade do sistema, a possibilidade de conseguir feedback por meio de formulários e comentários e a facilidade de obter dados de tempo de clique, erros e acertos. Além disso, a ferramenta ajudou no gerenciamento da pesquisa, indicando quantos usuários estavam realizando o teste, a cidade dos participantes e a média de tempo gasto para realizar a atividade.

Os resultados apresentados neste artigo trazem como principal contributo o relato de uma experiência positiva de testes remotos na área de design centrado no usuário, que pode ser uma alternativa para promover maior viabilidade técnica e econômica a pesquisas de empresas e instituições de ensino, que tenham como desafios as distâncias geográficas em relação aos usuários típicos e a diversidade destes grupos.

\footnotetext{
${ }^{2}$ Acesse os testes pelos endereços: Card sorting: <https://p00a1880.optimalworkshop.com/optimalsort/y4fl8d35> e Medicação de performance: <https://p00a1880.optimalworkshop.com/chalkmark/x64x6b07>
} 


\section{Referências}

ASSOCIAÇÃO AMAZONENSE DE MUNÍCIPIOS. Distâncias dos municípios em relação a capital. 2018. Disponível em: <http://portal.cnm.org.br/sites/8100/8133/Distancia_dos_Municipios _em_relacao_a_cap.pdf $>$. Acesso em: 15-03-2018.

FREIRE, L. L. Navegação e design em softwares educativos: uma abordagem ergonômica. Recife, 2005. 345 f. Tese (Doutorado) - Dissertação (Mestrado em Design) -Centro de Artes e Comunicação, Universidade Federal de Pernambuco, Recife, 2005.

GIL, A. C. Como elaborar projetos de pesquisa. [S.I.]: Atlas, 2002.

GOMES, Magno Federici. "Avaliação e natureza administrativa das instituições de ensino superior." Ensaio: Avaliação e Políticas Públicas em Educação 18.68 (2010).

IDEO. Design Kit: The Human-Centered Design Toolkit. 2009. Disponível em: <https://www.ideo.com/post/design-kit>. Acesso em: 13-06-2017.

KALBACH, J. Design de navegação web. Trad. de Eduardo Kessler Piveta. Porto Alegre: Bookman, 2009.

MARTINS, Anny Caroline Castelo Branco. Informatização dos processos do programa de monitoria da UFAM. Universidade Federal do Amazonas (2017).

MEC. Qual é a diferença entre faculdades, centros universitários e universidades? 2017. Disponível em: $\quad$ http://portal.mec.gov.br/pec-g/127-perguntas-frequentes-911936531/educacao-superior399764090/116-qual-e-a-diferenca-entre-faculdades-centros-universitarios-e-universidades> Acesso em: 15-06-2017.

NIELSEN, J. Heuristic evaluation. In Use-it.com: Jakob Nielsen's Web site. 2002. Disponível em: <http://www.useit.com>. Acesso em: 13-06-2017.

NORMAN, D. A. O design do dia-a-dia. [S.I.]: Rocco, 2006.

PIRAUÁ, J.; MOURA, D.; PADOVANI, S. Discutindo o card sorting: uma análise da técnica. 6o Congresso Internacional de Ergonomia e Usabilidade, Design de Interfaces e Interação HumanoComputador, 2006.

PROPLAN. UFAM em números. 2014. Disponível em: http://www.proplan.ufam.edu.br/Ufam-emnumeros-v3.pdf>. Acesso em: 15-03-2018.

SHIBUYA, L. d. N. Revista de Design de Interação. 2010. Disponível em: <http://designdeinteracao.com.br/wp/wp-content/uploads/2015/08/ revista_ed_03.pdf>. Acesso em: 20-06-2017.

TEIXEIRA, F. Introdução e boas práticas em UX Design. [S.I.]: Editora Casa do Código, 2015. 